\title{
Nonsense mutations at Arg-1947 in two cases of familial neurofibromatosis type 1 in Japanese
}

\author{
Takahiko Horiuchi ${ }^{1}$, Nobuaki Hatta ${ }^{1}$, Mitsuru Matsumoto ${ }^{1}$, Hisashi Ohtsuka ${ }^{2}$, Francis S. Collins ${ }^{3}$, \\ Yuzuru Kobayashi' ${ }^{1}$, Shigeru Fujita ${ }^{1}$ \\ ${ }^{1}$ First Department of Internal Medicine, Ehime University, Shigenobu, Ehime, 791-02 Japan \\ ${ }^{2}$ Department of Dermatology, School of Medicine, Ehime University, Ehime, 791-02 Japan \\ ${ }^{3}$ Howard Hughes Medical Institute, University of Michigan Medical Center, Ann Arbor, MI 48109-0650, USA
}

Received: 7 June 1993 / Revised: 3 August 1993

\begin{abstract}
We report two familial cases of NF1 presenting as $C$ to $T$ transitions changing an Arg-1947 codon to a stop codon. In one of the two families, cosegregation of the mutation with NF1 was demonstrated, indicating this mutation causes the disease in this family. As the same mutation at Arg- 1947 has been reported previously in three cases of unrelated Caucasians (two are sporadic; the origin of the other is not reported), the codon at Arg-1947 (CGA) in the NF1 gene is considered to be a hotspot common among different ethnic groups and also among familial and sporadic cases.
\end{abstract}

\section{Introduction}

Neurofibromatosis type 1 (NF1), also known as von Recklinghausen's neurofibromatosis, is an autosomal dominant disorder affecting about 1 in 4000 individuals in all ethnic groups. So far, few cases of NF1 have been reported presenting with abnormalities in the NF1 gene (reviewed by Gutmann and Collins 1993). In the present study, we describe nonsense mutations at Arg-1947 (numbering based on Marchuk et al. 1991) in two Japanese families shown to be unrelated by using a new polymorphic restriction fragment length polymorphism (RFLP) marker within the NF1 gene. We also show that the NF1 messages from the mutant allele and from the normal allele are transcribed in an Epstein-Barr (EB) virus-transformed lymphoblastoid cell line from one of the patients.

\section{Materials and methods}

\section{NFI patients}

Twenty-five unrelated Japanese NF1 patients were studied; 15 had a family history of the disease and 10 were sporadic.

Correspondence to: T. Horiuchi
Polymerase chain reaction/single-strand conformation polymorphism analysis

The experimental procedure has been described previously (Orita et al. 1989). DNA samples for single-strand conformation polymorphism (SSCP) analysis were generated by using the polymerase chain reaction (PCR) with the primer pairs for exon 31 , originally described as the primer pairs for "exon 4 " by Cawthon et al. (1990). The PCR products from patients showing abnormal bands were subjected to $1.2 \%$ agarose gel electrophoresis, purified on a SUPREC-01 column (Takara, Kyoto, Japan), reamplified by asymmetric PCR and directly sequenced by the dideoxy chain termination method.

\section{mRNA analysis}

Total RNA was prepared from an EB virus-transformed lymphoblastoid cell line of case 1 and was amplified by a pair of primers (5'GCAAATCTAGTATTGAATTG 3', nucleotides 57415760 , and the reverse primer 5'CCTTCCAATAACCTTGCTTG3', nucleotides 6077-6096) using the GeneAmp RNA PCR kit (Perkin Elmer Cetus, Norwalk, Conn.) according to the manufacturer's instructions. The product was amplified asymmetrically and directly sequenced.

\section{RFLP analysis}

DNA from patients or healthy individuals was digested with $E c o$ RI, $B g l I I$, or BamHI, and subjected to Southern blotting as described (Horiuchi et al. 1989). Seven overlapping NF1 cDNA clones (GE2, FF13, FF1, FB5D, AE25, P5, B3A) encompassing the entire coding region and part of the $5^{\prime}$ and $3^{\circ}$ untranslated regions of the NF1 gene were utilized as probes (Marchuk et al. 1991).

\section{Results and discussion}

RFLP analysis using EcoRI, $B g l \mathrm{II}$, or $B a m H I$ showed that there were no differences between NF1 patients and healthy individuals (data not shown). However, we found an EcoRI polymorphism that is detected by probe GE2, and that has been reported independently in Caucasians 


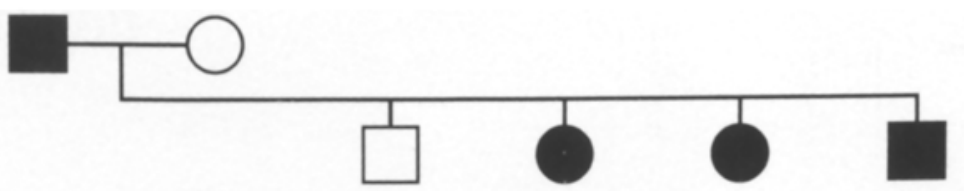

a)

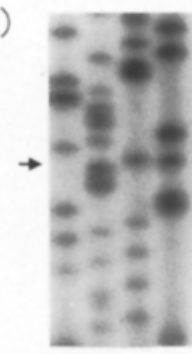

G A T C

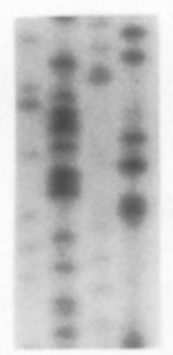

G A T C

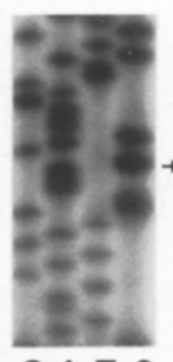

G A T C
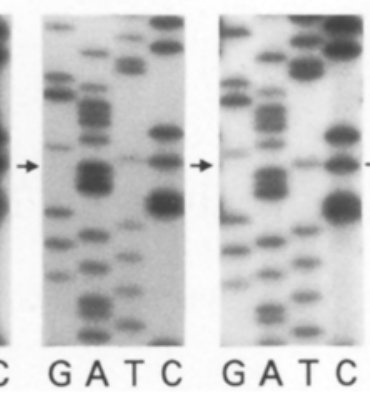

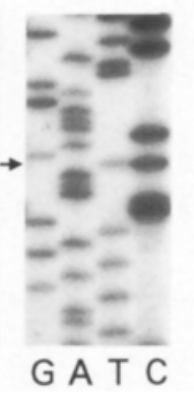

c)

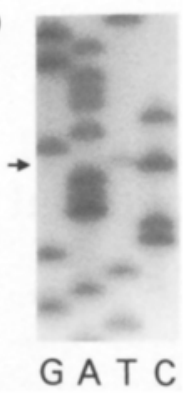

b)

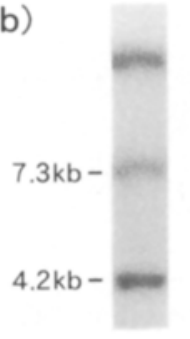

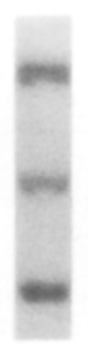

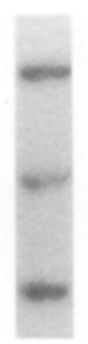

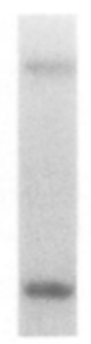

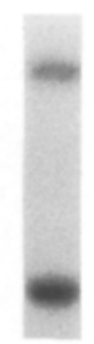

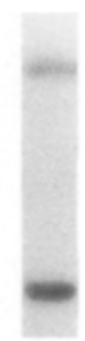

Fig. 1. Top Pedigree of the family of case 1 . a Direct genomic sequencing of exon 31 ; b EcoRI RFLP detected by probe GE2 (Marchuk et al. 1991); c direct sequencing of the RT-PCR product of exon 31 from a lymphoblastoid cell line of one patient. The location of the mutation in $\mathbf{a}$ and $\mathbf{c}$ is denoted by arrows by Reyniers et al. (1993). The frequency of the two-allele polymorphism with bands at $7.3 \mathrm{~kb}$ (A1) and $4.2 \mathrm{~kb}$ (A2) were 0.48 and 0.52 , respectively, when estimated from 80 unrelated Japanese individuals.

In order to find more subtle abnormalities in the NF1 gene, we performed PCR-SSCP analysis of exon 31. Two out of 25 patients (cases 1 and 17) showed bands of altered mobility. The nucleotide sequence of patient DNA revealed the transition of the codon CGA for Arg-1947 to the stop codon TGA in both cases. Case 1 was a 40 -yearold male and case 17 was a 25 -year-old female, both of whom had a family history of the disease and presented with multiple neurofibromas and cafe-au-lait spots. Other abnormalities, such as malignant tumors, were not found in either of the two cases or members of their families. All other family members of case 1 , including both parents and three siblings, were studied for the $\mathrm{C}$ to $\mathrm{T}$ transition at ARG-1947 to confirm that this mutation is responsible for their NF1 (Fig. 1a). The perfect coinheritance of the nonsense mutation at Arg-1947 and NF1 in case 1 family indicates the importance of the mutation for the pathogenesis of NF1. The affected siblings of case 1 showed homozygosity for $\mathrm{A} 2$, whereas the father was a heterozygote for A1 and A2 (Fig. 1b). This indicates that the mutation is on the A2 allele. On the other hand, case 17 is homozygous for A1 (data not shown), indicating that the mutation is on the A1 allele and that the two families are unrelated. Although the same mutation has been reported independently in three cases of NF1 (Cawthon et al. 1990; Estivill et al. 1991; Ainworth et al. 1993), our cases are the first described in an ethnic group other than Caucasians. In addition, the present study is the first report describing fa- milial NF1 cases presenting with the nonsense mutation, because two out of the previously reported cases with the same mutation were sporadic and the origin of the other case was not reported. The $\mathrm{C}$ at the codon for Arg-1947 (CGA) is the only hotspot so far detected in the NF1 gene. The frequency of this mutation in our Japanese NF1 patients is $8.0 \%$ ( 2 in 25 patients), whereas that in Caucasians is $1.1 \%$, estimated from the data from the previous three reports (3 in a total of 158 patients) and that of Upadhyaya et al. (1992) (none in 110 patients). Further study should clarify whether the frequency of this mutation at Arg-1947 is different among different ethnic groups.

Finally, we showed that the NF1 gene on the normal and mutant allele are both transcribed in a lymphoblastoid cell line derived from case 1 (Fig. 1c). If no other mutations reside in the coding region of the mutant allele, the mutant message encodes abnormal neurofibromin that lacks its carboxy one third (Arg-1947 to Val-2818), but that still preserves the GTPase-activating protein-related domain (GRD). It will be of great interest to study the biochemical and biological properties of this mutant neurofibromin in order to understand the structure-function relationships of native neurofibromin.

Acknowledgements. We would like to thank Dr. David H. Gutmann for helpful discussions and his review of the manuscript.

\section{References}

Ainworth PJ, Rodenhiser DI, Costa MT (1993) Identification and characterization of sporadic and inherited mutations in exon 31 
of the neurofibromatosis (NF1) gene. Hum Genet 91:151156

Cawthon RM, Weiss R, Xu G, Viskochil D, Culver M, Stevens J, Robertson M, Dunn D, Gesteland R, O'Connel P, White R (1990) A major segment of the neurofibromatosis type 1 gene: cDNA sequence, genomic structure, and point mutations. Cell 62:193-201

Estivill X, Lazaro C, Casals T, Ravella A (1991) Recurrence of a nonsense mutation in the NF1 gene causing classical neurofibromatosis type 1 . Hum Genet $88: 185-188$

Gutmann DH, Collins FS (1993) The neurofibromatosis type 1 gene and its protein product, neurofibromin. Neuron 10:335343

Horiuchi T, Macon KJ, Kidd VJ, Volanakis JE (1989) cDNA cloning and expression of human complement component $\mathrm{C} 2$. J Immunol 142:2105-2111
Marchuk DA, Saulino AM, Tavakkol R, Swaroop M, Wallace MR, Andersen LB, Mitchell AL, Gutmann DH, Boguski M, Collins FS (1991) cDNA cloning of the type 1 neurofibromatosis gene: Complete sequence of the NF1 gene product. Genomics 11:931-940

Orita M, Suzuki Y, Sekiya T, Hayashi K (1989) Rapid and sensitive detection of point mutations and DNA polymorphisms using the polymerase chain reaction. Genomics 5:874-879

Reyniers E, Boulle K de, Marchuk DA, Andersen LB, Collins FS, Willems PJ (1993) An EcoRI RFLP detected by the human NF1 gene. Hum Genet (in press)

Upadhyaya M, Shen M, Cherryson A, Farnham J, Maynard J, Huson SM, Harper PS (1992) Analysis of mutations at the neurofibromatosis 1 (NF1) locus. Hum Mol Genet $1: 735-740$ 